\title{
LA RACE GATINAISE \\ ÉTUDE DE SA VARIABILITÉ GENÉTIQUE \\ EN VUE D'OBTENIR DES CROISEMENTS HOMOGÈNES
}

PAR

P. MERAT

Station de Recherches Avicoles

C.N.R.Z., Jouy-en-Josas (S.-et-O.).

\section{PLAN DU MEMOIRE}

Introduction : But des analyses.

I. - Organisation des analyses.

II. - Présence et fréquence de certains allèles.

I. - Blanc récessif $c$.

2. - Blanc dominant $I$.

3. - Argenté et doré liés au sexe : $S$ et $s$. Quelques manifestations particulières du doré.

4. - Série allélique $E, e, e^{+}$.

5. - Le "blue " $G$.

6. - Barrure liée au sexe : $B$.

7. - - Patte jaune $\%$.

8. - Emplumement lié au sexe.

III. - Observations sur certains phénotypes particuliers.

I. - Pigmentation brune. Le " saumon ".

2. - Coloration retardée.

3. - Patte grise ou bleutée.

IV. - Conclusions.

I. - Génotype de la Gâtinaise.

2. - Programme de sélection relatif aux gènes étudiés.

\section{INTRODUCTION : BUT DES ANALYSES}

Par ces analyses, nous avons voulu nous assurer du génotype de la souche Gâtinaise que nous possédons à la station, en vue de rendre cette souche homozygote pour certains gènes.

JULL ( 18 ) définit un cryptomère comme un caractère dont le gène responsable est présent, mais qui reste invisible, parce que d'autres gènes, nécessaires à son expression, manquent dans le génotype jon 
désigne couramment par cryptomère, non le caractère, mais le gène correspondant). Ainsi, LIPPINCOTT (26), CREW (IO), QUINN (29) et JFFFREY (I6) ont analysé la Wyandotte pour certains cryptomères, ainsi que Cochez (7). Jefrrey analyse également plusieurs souches de Plymouth Rock blanche. LEGRAND (25) analyse la Malines blanche.

Or, l'homozygotie de la Gâtinaise pour certains cryptomères, masqués en race pure par l'absence de pigmentation, est indispensable si l'on veut obtenir avec cette race des croisements de première génération homogènes. Elle est nécessaire aussi, avec l'absence de blanc dominant $I$, à la création d'une souche $c c$ ii ss, qui serait blanche comme la Gâtillaise, et susceptible en outre de donner certains croisements sexables à la naissance.

Nous avons donc cherché à reconnaître la présence, et la fréquence, de certains cryptomères intéressant la couleur du duvet, du plumage ou des pattes. Secondairement, des manifestations particulières de certains gènes ont été observées au cours des croisements effectués, et feront 1'objet d'études ultérieures.

\section{I. - ORGANISATION DES ANALYSES}

L'analyse a été limitée à des $\varnothing$ Gâtinaises, sauf un test préalable portant sur deux $O^{x}$. Le duvet a été examiné à la naissance, le plumage et la couleur des tarses à 8 semaines, ce qui a permis d'étudier les relations entre certains duvets et certains plumages. Le sexe a pu être identifié sur tous les animaux à partir de I954, mais pas en automne I953. L'analyse a été limitée, en général, aux mères ayant donné 7 enfants au moins relativement à un caractère donné.

Les croisements suivants ont été faits successivement:

a) Analyse préalable : $20^{\Upsilon}$ Gâtinais croisés chacun avec Io $Q$ Sussex au printemps 1953 .

b) Ia première analyse d'automne I953 comportait 6 parquets pedigrees avec aut total 6I $q$ Gâtinaises. I es $\sigma^{x}$ utilisés étaient issus d'un croisement de première génération $\left(O^{*}\right.$ Marans coucou argenté $\times q$ Sussex). D'après les races parentes et le phénotype de ces coqs, leur génotype était le suivant : ii $E e B b$, et probablement $C C$. Ceci est confirmé par 1e fait que ces $\sigma^{*} n^{\prime}$ ont donné que des enfants colorés (présence de pigment dans le plumage) avec les $2 / 3$ des $q$ Gâtinaises, lesquelles étaient $c c$ (comme il est montré plus loin). En outre, ces $\sigma^{x}$ étaient $S S$ ou $S s$, et homozygotes pour la patte blanche $(W W)$ et pour l'absence de "blue " ou dilution du noir (c'est-à-dire $g g$, ou $b l \quad b l$ suivant la notation de HUTT (I3)).

Ces $\sigma^{\prime}$, seuls disponibles pour cette première analyse, ont permis 
de tester les $q$ Gâtinaises pour le blanc dominant $I$, et divers autres cryptomères.

c) La deuxième analyse d'automne I953 comportait Io parquets pedigrees, avec au total I05 + Gâtinaises et ro $0^{\top}$ Rhode Island rouges.

Ces deux analyses d'automne ont comporté chacune 3 éclosions. Au total, 243 poussins pedigrees ont été décrits dans la première série, et $38 \mathrm{I}$ dans la deuxième.

d) Au printemps 1954, 6 parquets pedigrees ont été constitués chacun avec I $0^{x}$ R.I.R. et Io $\bar{q}$ Gâtinaises, dont certaines avaient déjà reproduit à l'automne. 622 poussins, issus de 6 éclosions, ont été étudiés.

e) A l'automne I954, 20 parquets d'analyse de I coq et Io poules ont permis de suivre diverses ségrégations du croisement $o^{x}$ R.I.R. $\times q$ Gâtinaise. Certains parquets étaient des II $\times$ FI, d'autres des croisements de retour $\sigma^{x}$ R.I.R. $\times q$ FI. Dans d'autres encore, des $\sigma^{x}$ ou o FI étaient croisés à des $q$ ou $\sigma^{*}$ issus de croisements multiples (Rhode $\times$ Wyandotte $) \times$ (Gâtinais $\times$ Sussex). Quoique ces derniers parquets n'aient pas été faits en vue de la présente analyse, ils fournissent pour celle-ci un certain nombre de renseignements.

f) Enfin, au printemps 1955, I5 parquets de constitution analogue à ceux de l'automne 1954 ont permis de compléter l'étude de la Fr.

\section{II. - PRESENCE ET FREQUeNCE de Certains ALLÈLES}

\section{1. - Blane « récessif " $e$.}

I a majorité des q Gâtinaises a donné, en première génération, uniquement des enfants colorés (présence de pigment dans le plumage). C'est également le cas du $\sigma^{\top} 922$, testé au printemps 1953.

L'accouplement FI $\times$ FI d'animaux issus de ces $q$ fait apparaitre des descendants blancs, dans une proportion qui ne diffère pas significativement de 3 colorés/I blanc.

D'autres croisements de divers types ont été réalisés. Ils incluaient des $\sigma^{x}$ et des $q$ FI $\left(\sigma^{x}\right.$ R.I.R. $\times q$ Gâtinaises). I,eurs partenaires étaient soit des R.I.R. purs, soit des animaux issus du croisement quadruple (R.I.R. $\times$ Wyandotte) $\times$ (Gâtinais $\times$ Sussex), les parents des 3 races R.I.R., Wyandotte et Sussex étant de génotype contrôlé pour 1a coloration.

$I_{1}$ es faits énoncés plus haut conduisent à penser que toutes les $q$ Gâtinaises analysées portaient un gène récessif autosomal empêchant toute pigmentation dans le plumage, et l'ensemble des résultats, comprenant des croisements avec des descendants de Wyandottes, est conforme à l'hypothèse de l'identité de ce gène avec celui porté par les Wyandottes. (Tableau $n^{0}$ I). Nous admettrons que ce gène est $c$ (I3). 
TABLEAA I

Résultats $F 2$ relatits au blanc récessit.

\begin{tabular}{|c|c|c|c|c|}
\hline $\begin{array}{l}\text { Génotype supposé } \\
\text { des parents }\end{array}$ & $\begin{array}{l}\text { Proportions théoriques } \\
\text { dans la descendance }\end{array}$ & Proportions observées & $x^{2}$ & \\
\hline $\begin{array}{l}\mathrm{Cc} \times \mathrm{Cc}: \\
\text { Automne } 5954 \ldots \ldots \ldots \\
\text { Printemps } 1955 \ldots \ldots \cdots \\
\text { Total } \ldots \ldots \ldots \ldots\end{array}$ & $\begin{array}{l}3 \text { colorés/r blanc } \\
- \text { id }^{0}-\end{array}$ & $\begin{array}{cc}77 \mathrm{col} / 16 \mathrm{bl} \\
485 & / 186 \\
74^{2} & / 258\end{array}$ & $\begin{array}{l}3,0 \mathrm{P} \text { 非 } \mathrm{o}, \mathrm{10}] \\
0,9 \\
0,3\end{array}$ & $\begin{array}{l}\text { N. S, } \\
\text { N. S. } \\
\text { N. S. }\end{array}$ \\
\hline $\begin{array}{l}\mathrm{CC} \times \mathrm{Cc}: \\
\text { Automne I954 } \ldots \\
\text { Printemps I } 955 \ldots\end{array}$ & $\begin{array}{l}\text { Tous colorés } \\
- \text { id }^{0}-\end{array}$ & $\begin{array}{l}\text { Tous colorés } \\
- \text { ido - }\end{array}$ & & \\
\hline 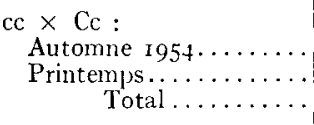 & $\begin{array}{l}\text { I coloré/I blanc } \\
\text { - ido }\end{array}$ & $\begin{array}{l}139 / 124 \\
21 / 18 \\
160 / 142\end{array}$ & $\begin{array}{l}0,9 \\
0,2 \\
I, I\end{array}$ & $\begin{array}{l}\text { N. S. } \\
\text { N. S. } \\
\text { N. S. }\end{array}$ \\
\hline
\end{tabular}

Le blanc dominant $I$ n'est que très partiellement répandu chez nos Gâtinaises, comme nous le verrons plus loin. L,e fait qu'en plusieurs années, nous n'ayons jamais observé une seule Gâtinaise colorée confirme encore que toutes portent le blanc récessif.

\section{2. - Blane « dominant »I.}

Dans la descendance de certaines poules, sont apparus en première génération deux types d'animaux : les uns entièrement blancs, le pigment noir étant absent ou réduit à quelques petites taches; les autres blancs avec des taches rouges ou brunes d'étendue variable. De tels phénotypes suggèrent la présence $d u$ blanc dominant $I$. Pour abréger, nous avons par la suite appelé "blancs " les animaux du type cité, c'est-à-dire sans pigment noir, et "colorés " ceux avec pigment noir.

Tous les $O^{*}$ employés étant $i i$, rappelons pour mémoire les possibilités qui se présentaient suivant le génotype de la $q$ :

1er cas: $O I i:$ Il peut y avoir des descendants colorés et des descendants blancs.

2 e cas: ㅇ $I I$ : Tous les descendants sont blancs (ou du moins sans pigment noir).

3e cas: $\subsetneq$ ii: Tous les descendants ont du pigment noir.

Une mère a été supposée $i i$ si elle a donné au moins 7 descendants, tous colorés. La probabilité pour qu'elle soit $I i$ est en effet dans ce cas inférieure à I p. Ioo. De même, une mère n'a été supposée $I I$ que si elle a donné au moins 7 enfants, tous sans pigment noir. C'est pourquoi, ceci étant valable pour tous les gènes, seules ont été considérées en général dans les analyses les mères ayant donné 7 enfants au moins. 
Chez les $q$ ayant donné en FI des enfants blancs et des enfants colorés, les proportions suivantes ont été observées au printemps I954:

\section{TABLEAU II}

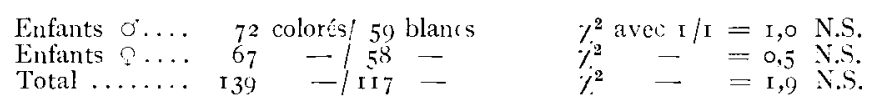

Dans les analyses antérieures, les ségrégations paraissaient aberrantes :

\section{TABLEAU III}

\begin{tabular}{|c|c|}
\hline & \\
\hline 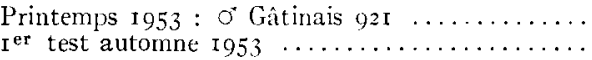 & $\begin{array}{l}1.3 \\
76\end{array}$ \\
\hline $2^{\text {eme }}$ test autonne $\mathrm{r} 953 . \ldots \ldots \ldots \ldots \ldots \ldots$ & 122 \\
\hline
\end{tabular}

Les résultats ultérieurs $\left(\mathrm{F}_{2}\right)$ ont montré que des erreurs de classification s'étaient produites sur les descendants issus de quelques mères. Comme il est indiqué plus loin, $G$, dilueur du noir (I3), est répandu chez la Gâtinaise. Dans les deux tests d'automne I953, certaines mères ii $G g$ ont donné des enfants également $G g$, dont la plupart ont été classés comme "colorés", mais quelques-uns comme "blancs". Ceci doit suffire à expliquer le défaut apparent d'animaux blancs en I953, chez les mères supposées $I i$. La présence chez quelques mères de $B$, indiquée plus loin, a pu parfois encore atténuer la pigmentation des parties normalement noires et produire des cas de "blanc artificiel " analogues à ceux signalés par Cochez (7) sur la Marans blanche.

En effet, quelques $\varnothing \mathrm{FI}$ supposées $I i$ ont été accouplées à des $\sigma^{*}$ $C C$ ii. Quatre d'entre elles n'ont donné que des enfants colorés, certains portant $G$. Ces $q$ descendaient précisément de 3 mères qui n'avaient eu chacune qu'un ou deux enfants classés "blancs " et tous les autres colorés.

De même, la F2 donnée par quelques descendants du $\sigma^{x} 92 \mathrm{I}$ indique qu'il était $I i G g$, et l'excès de "blancs" dans sa descendance FI s'explique également par le classement d'animanx $G g$ dans la catégorie "blancs ". Ceci est confirmé par le fait que quelques-unes de ses filles, classées "blanches", se sont comportées en F2 comme des ii.

Pour les autres $q$ FI supposées $I i$ et accouplées à des $\sigma^{\top} C C i$, les proportions observées ont été les suivantes:

\section{TABLEAU IV}

Automne $195+\ldots \ldots \ldots \ldots$

Printem 1 s $1955 \quad \ldots \ldots \ldots \ldots$ Total .

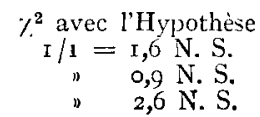


Ces proportions sont conformes à l'hypothèse d'un gène unique autosomal dominant, qui présente toutes les caractéristiques de $I$.

Le nombre d'animaux portant à la fois $I$ et $c$ en F2 était trop faible pour vérifier la proportion 3 colorés $/ 5$ blancs dans le croisement $C c$ ii $\times$ Cc Ii.

\section{Proportion de $I$ et $i$ dans la génération parentale}

En tenant compte des quelques erreurs de classification initiales qui avaient été faites, les diverses analyses ont donné les résultats suivants :

\section{TABLEAU V}

\begin{tabular}{|c|c|c|c|c|}
\hline & $\left\{\begin{array}{c}\text { Nombre de } \\
\text { analysées } \\
\text { avec certitude }\end{array}\right.$ & \& II & f Ii & $q \mathrm{it}$ \\
\hline 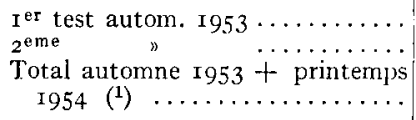 & $\begin{array}{l}23 \\
35 \\
105\end{array}$ & $\overline{-}$ & $\begin{array}{l}17 \\
27 \\
61\end{array}$ & $\begin{array}{r}6 \\
12 \\
44\end{array}$ \\
\hline
\end{tabular}

A partir des génotypes identifiés avec certitude, nous obtenons l'estimation la plus vraisemblable des fréquences respectives des allèles $I$ et $i$ chez les mères d'après la loi de HARDy, l'hypothèse de la panmixie étant ici vérifiée (Les reproductrices Gâtinaises ont bien été toujours accouplées au hasard en ce qui concerne les allèles $I$ et $i$ ) :

$$
\mathrm{P}_{i}=7 \mathrm{I} \% \quad q_{\mathrm{I}}=29 \%
$$

chiffres voisins des chiffres partiels trouvés en automne :

$$
\begin{array}{lll}
\mathrm{I}^{\mathrm{er}} \text { test } & p_{i}=63 \% & q_{\mathrm{I}}=37 \% \\
2^{\mathrm{e}} \text { test } & p_{i}=70 \% & q_{\mathrm{I}}=30 \%
\end{array}
$$

\section{3. - Argenté et doré lié au sexe : S et s.}

Au printemps I953, les deux $\sigma^{x}$ Gâtinais employés n'ont donné que des enfants du type "argenté ". Ils devaient donc être SS.

A l'automne I953, peu de conclusions pouvaient être tirées du $\mathrm{I}^{\mathrm{er}}$ test quant à la présence de l'argenté et du doré chez la Gâtinaise ; et, de toutes façons, le sexe n'a pu être déterminé chez tous les animaux. Cependant, 2 mères ont donné, chacune, un coquelet rouge herminé, ss selon toutes les apparences. Ces deux mères étaient $s^{-}$selon toute vraisemblance. 
Au printemps r954, le sexe a pu être connu pour tous les animaux. Parmi les animaux colorés, ont été observés :

I2 $\sigma^{x}$ de type "doré ",

$25^{8} o^{x}$ argentés,

243 q dorées (le duvet étant d'une teinte rouge assez variable).

Parmi les animaux $I i$, ont été observés :

$38 o^{x}$ tout blancs,

$24 \sigma^{t}$ avec quelques taches brunes (cf. p. I7),

58 o blanches avec plus ou moins de "doré ".

Les I2 $\sigma^{x}$ dorés sont issus de 2 mères, qui n'ont donné aucun $\sigma^{x}$ argenté. Toutes les autres mères ont donné uniquement des $\sigma^{x}$ argentés.

La Fi est conforme à 1'hypothèse suivant laquelle la majorité des q Gâtinaises portaient un gène lié au sexe, produisant la couleur argentée chez les fils, et il est raisonnable de penser qu'il s'agit de $S$, allèle de $s$ porté par les $\sigma^{\top}$ R.I.R. L'allèle $s$ apparaît peu répandu chez nos Gâtinaises.

Tant en automne 1954, qu'au printemps 1955, divers types de croisements de $2^{e}$ génération ont été faits, incluant FI $\times$ Fr et divers croisements d'animaux FI avec des produit du croisement multiple (Rhode $\times$ Wyandotte $) \times$ (Gâtinais $\times$ Sussex $)$, de génotype connu ou présumé, relativement à $S$ et $s$.

Pour mémoire, les résultats des divers types de croisements ont été les suivants :

\section{'TABleau VI}

Résultats F2 relatifs à $\mathrm{S}$ et $\mathrm{s}$ (sur les animantx ii).

\begin{tabular}{|c|c|c|c|c|c|c|}
\hline \multirow{2}{*}{$\begin{array}{l}\text { Génotype } \\
\text { présumé des } \\
\text { parents }\end{array}$} & \multicolumn{2}{|c|}{ Fils } & \multirow[b]{2}{*}{$\gamma^{2}$} & \multicolumn{2}{|c|}{ Filles } & \multirow{2}{*}{$\check{1}^{2}$} \\
\hline & $\begin{array}{l}\text { Proportion } \\
\text { attendue }\end{array}$ & $\begin{array}{l}\text { Proportion } \\
\text { observée }\end{array}$ & & $\begin{array}{l}\text { Proportion } \\
\text { attendue }\end{array}$ & $\begin{array}{l}\text { Proportion } \\
\text { observée }\end{array}$ & \\
\hline $\begin{array}{l}\mathrm{SS} \times \mathrm{S}^{-} \ldots \ldots \\
\mathrm{SS} \times \mathrm{S}^{-} \ldots \ldots \ldots\end{array}$ & Tous argentés & Tous argentés & & Toutes argent. & Toutes argent. & \\
\hline $\mathrm{Ss} \times \mathrm{s}^{-}$ & $\begin{array}{l}\text { I argenté } \\
\text { /1 doré }\end{array}$ & $\begin{array}{l}229 \text { argentés } \\
\text { /201 dorés }\end{array}$ & $\begin{array}{l}2,1 \\
\text { N.S }\end{array}$ & $\begin{array}{l}\text { I argentée } \\
\text { I dorée }\end{array}$ & $\begin{array}{l}276 \text { argentées } \\
1245 \text { dorées }\end{array}$ & $\stackrel{1,8}{N} . \mathrm{S}$ \\
\hline $\mathrm{ss} \times \mathrm{s}^{-} \ldots$ & Tous dorés & Tous dorés & & Toutes dorées & Toutes dorées & \\
\hline
\end{tabular}

Ces résultats confirment ceux observés en $\mathrm{Fr}$. On peut noter, dans le croisement $S s \times s^{-}$, un certain défaut d'animaux dorés ( $\sigma^{t}$ et $q$ réunis : 505 argentés/446 dorés). Cependant, ce défaut n'est pas significatif $\left(\%^{2}=3,7 \cdot 0, I>p>0,05\right)$. 


\section{Quelques manifestations particulières du doré :}

a) MANIFESTAtion DE S EN PRÉSENCE DE E : “ FACE ROUSSE ».

Dans le premier test d'automne I953, certains poussins au noir étendu présentaient une "face rousse ", déjà observée par Cochez (7) sur divers croisements, et par Boyer (4) sur des Marans coucou argenté, pures ou en croisement.

Les types de plumages correspondant à ces duvets noirs à face rousse indiquent qu'il s'agissait d'animaux $E e$ ou $E e^{+}$(cf. p. II-I2).

Rien ne contredit dans ces résultats 1'hypothèse suivant laquelle, dans le cas présent, $s$ produirait cette "face rousse", en présence de $E$ à simple dose. HuTT (I3) (p. I94), indique que, dans certains croisements $\sigma^{\pi}$ R.I.R. ou New-Hampshire $\times$ \& Plymouth Rock barrée, certaines mères étaient $E e$. On obtenait dans leur descendance diverses catégories, en particulier des $q b^{-} s-E e$, dont le duvet était noir, avec souvent une tache rouge sur la tête, rouge qui n'apparaissait pas chez les ơ $B b S s E e$.

Cette hypothèse pourrait être vérifiée ultérieurement par le croisement d'individus à "face rousse ) avec des R.I.R. par exemple, porteurs de $s$.

b) MANifESTATION DE S EN PRÉSENCE DE $\dot{I} i$.

Cette manifestation est variable. L'extension maximum du rouge donne des animaux entièrement rouges, sauf les parties normalement noires dans le type herminé, parties remplacées par du blanc. Ceci rejoint les observations de Cock et PEAsE (9).

Comme nous le verrons plus loin, d'autres facteurs que l'allèle $s$ peuvent donner une coloration rougeâtre ou brune sur le plumage $I i$.

Les duvets des animaux dorés hétérozygotes pour le blanc dominant sont également variables et vont du blanc presque total à un rouge presque complètement étendu.

c) MANifESTATION DE $S$ EN PRÉ.SENCE DE $c c$.

Les croisements dont une partie est analysée ici ayant pour but d'obtenir une souche de constitution génétique contrôlée, qui serait en particulier $c c$ ss pour les $\sigma^{x}, c c s^{-}$pour les $q$, il serait intéressant de pouvoir détecter phénotypiquement au moins une partie des animaux ayant cette constitution, ce qui réduirait d'autant le nombre d'individus à tester sur la descendance.

PunNetT, cité par Hutt (I3), trouve du pigment rouge chez des descendants $F_{2}$ d'un croisement de "Silkies " qu'il suppose porter un gène de blanc récessif. Cependant, la nature génétique de ce blanc ne paraît pas avoir été complètement éclaircie. QUINN (29) et JEFFREY (I6), analysant les cryptomères de souches de Wyandotte blanche, trouvent $s$ 
chez certains animaux, et ne mentionnent pas de différences phénotypiques causées par $S$ ou $s$ chez les $c c$.

JEFFREY (I6) trouve des duvets à teinte rosée chez des animaux cc $S S$ ou $S^{-}$. Cette teinte n'est donc pas dûe ici à $S$. Kimball (I9), (20) note que du "pile " peut se rencontrer chez des blancs récessifs, non dû̀ à $s$.

Les données présentes ont vérifié que beaucoup d'animaux $c c$ et porteurs de $s^{-}$étaient entièrement blancs, sans aucune trace décelable de rouge. Chez certains, on peut observer de petites taches rouges ou une teinte rosée dans le duvet à I jour ; le plumage peut aussi comporter de petites taches rouges de teinte rappelant $s$, le plus souvent à la naissance du bec ou sur le crâne, plus rarement ailleurs. Le tableau suivant montre que 1'on ne trouve en fait ces taches que dans les croisements pouvant comporter des descendants $s s$ ou $s^{-}$(et peut-être plus rarement $S s)$.

\section{TABLEAU VII}

Manifestation de s en présence de cc.

\begin{tabular}{|c|c|c|c|c|c|}
\hline & \multirow[b]{2}{*}{$\begin{array}{l}\text { Génotype des } \\
\text { parents }\end{array}$} & \multicolumn{4}{|c|}{ Phénotype des enfants } \\
\hline & & $\begin{array}{l}\text { Duvet blanc. } \\
\text { Plumage blanc }\end{array}$ & $\begin{array}{l}\text { Duvet à taches } \\
\text { roses ou rouges. } \\
\text { Plumage blanc }\end{array}$ & $\begin{array}{l}\text { Duvet blanc. } \\
\text { Plumage taché } \\
\text { rouge }\end{array}$ & $\begin{array}{l}\text { Duvet à taches } \\
\text { roses ou rouges. } \\
\text { Plumage taché } \\
\text { rouge }\end{array}$ \\
\hline \multirow{3}{*}{ Fils } & $\begin{array}{r}\mathrm{SS} \times \mathrm{S}^{-} \ldots \ldots \\
-\times \mathrm{s}^{-} \ldots \ldots\end{array}$ & $\begin{array}{r}6 \\
29\end{array}$ & & 2 & \\
\hline & $\begin{array}{l}\text { Ss } \times s^{-} \ldots \ldots \\
-\times s^{-} \ldots \ldots\end{array}$ & $\begin{array}{l}32 \\
67\end{array}$ & I & $\begin{array}{l}2(?) \\
7\end{array}$ & 4 \\
\hline & $\begin{array}{l}\mathrm{ss} \times \mathrm{s}^{-} \ldots \ldots \\
-\times \mathrm{s}^{-} \ldots \ldots\end{array}$ & $\begin{array}{l}3 \\
\mathbf{I}\end{array}$ & & $\begin{array}{l}I \\
2\end{array}$ & $\mathbf{I}$ \\
\hline Filles & $\left\{\begin{aligned} \text { Père } & \text { SS } \ldots \ldots \\
& \text { Ss } \ldots \ldots \\
& \text { ss } \ldots \ldots\end{aligned}\right.$ & $\begin{array}{r}29 \\
\mathrm{II} 4 \\
\quad \mathrm{I}\end{array}$ & 6 & $\begin{array}{r}25 \\
7\end{array}$ & $\begin{array}{l}4 \\
1\end{array}$ \\
\hline
\end{tabular}

Quelques-uns des animaux observés ont d'autre part servi de reproducteurs. La correspondance entre phénotype (plumage) et génotype, indiqué par leur descendance, est la suivante :

TABLEAU VIII

\begin{tabular}{|c|c|c|}
\hline \multirow{2}{*}{ Génotype } & \multicolumn{2}{|c|}{ Phénotype (plumage) } \\
\hline & Tout blanc & Petites taches rouges \\
\hline 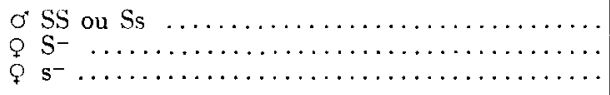 & $\begin{array}{r}11 \\
5 \\
18\end{array}$ & $\frac{-}{3}$ \\
\hline
\end{tabular}


I1 semble donc bien que les animaux présentant de petites taches rouges dans le plumage, surtout sur la tête, ou dans le duvet, soient pour les $Q$, des $s^{-}$; pour les $\sigma^{x}$, des ss (ou à la rigueur peut-être des $S s$ ). Cette observation peut permettre d'utiliser quelques-uns des animaux à blanc récessif sans avoir fait au préalable un test de descendance sur eux pour $S$ ou $s$.

Par contre, la présence d'une teinte rappelant le "saumon " ou le "brun " trouvé chez certaines o $C C S^{-}$ou $C c S^{-}$, n'est pas un reflet de $s$. Les reproductrices $c c$ observées pour ce caractère ont donné les résultats suivants (cf. p. I9).

TABLEAU IX

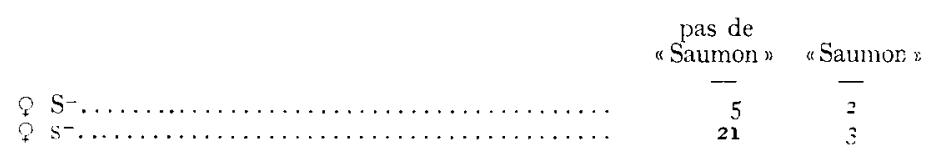

d) Conclusion.

Il apparaît donc qu'en présence de $I i$, ou de $c c, s$ peut se manifester, de façon variable, et que l'on peut reconnaitre sur l'apparence au moins une partie des animaux le portant. Mais, pour être sûre, cette reconnaissance doit encore exclure la confusion avec les taches brunes, ou " saumon ", de localisation particulière et d'aspect différent, qui peuvent se manifester également dans de nombreux génotypes (cf. p. I7), y compris cc comme nous venons de le voir.

\section{4. - Série allélique $\mathrm{E}, \mathrm{e}, \mathrm{e}^{+}$.}

a) Absence de $E$.

Le premier test d'automne I953 n'offrait que des possibilités restreintes d'analyse relativement à ces allèles. Les $\sigma^{x}$ étant hétérozygotes $E e$, on devait observer moitié d'enfants à noir étendu et moitié d'enfants à noir restreint dans l'hypothèse (vérifiée dans toutes les autres analyses) où aucune $P$ Gâtinaise ne portait $E$. Dans l'hypothèse contraire, la proportion de $E$ aurait dû être plus grande. En fait, la proportion observée chez les animaux non $I i$ a été de 80 à noir étendu/I23 à noir restreint, ce qui fait apparaître un défaut significatif de $\mathrm{E}\left(\%^{2}=9\right.$, I d'où $p<0$,or $)$. Ce défaut est conforme aux observations de Bernien et Cooney (3) et d'autres auteurs cités par eux, tendant à prouver que, d'une manière générale, une pigmentation plus importante du duvet s'accompagne de besoins plus grands de l'embryon en riboflavine, et, par suite, d'un pourcentage d'éclosion inférieur. Le défaut d'animaux dorés dans les ségrégations $S / s$, vu plus haut, est d'ailleurs également conforme à cette hypothèse. Ici même, BOYER (4) a trouvé lui aussi un défaut semblable 
d'animaux à noir étendı dans des croisements faits à partir de la Marans Coucou argentée.

Au deuxième test d'automne I953, et au printemps I954, avec des $\sigma^{\top}$ R.I.R. de génotype $e e$, aucune $\emptyset$ Gâtinaise ne s'est révélée porteuse du noir étendu $E$.

b) MANifEstation DE $e^{+}$.

Sur le duvet de certains descendants non $I i$, soit dans le croisement avec les $\sigma^{r} E e$, soit avec les $\sigma^{*}$ R.I.R., une bande brune ou grise, très variable, sur la tête et le dos, parfois encadrée de deux bandes plus minces, reproduisant le duvet rayé ou " stripe ", suggère la présence de l'allèle $e^{+}$(I9), (20) (ou à la rigueur d'un des allèles d'effet voisin prévus par Kimbali (23)).

A 8 semaines, au I $^{\text {er }}$ test d'automne, certains animaux, apparemment des $\sigma^{x}$, présentaient un plumage noir incomplètement étendu, avec des zones blanches (rappelant un $\sigma^{x}$ Faverolles) ou dorées (rappelant un $\sigma^{x}$ Leghorn doré). D'autres plumages rappelaient aussi $e^{+}$, de façon moins caractéristique.

Dans la FI $̛^{x}$ R.I.R. $\times q$ Gâtinaise, un duvet à rayure plus ou moins nette s'accompagnait souvent par la suite d'un sous-plumage plus ou moins foncé dans la région dorsale.

Cet allèle $e^{+}$, ou d'effet voisin de $e^{+}$, apparaît assez répandu chez la Gâtinaise. En effet, nous pouvons admettre que les génotypes des mères ayant donné au moins 6 enfants colorés en croisement avec des $\sigma^{T}$ R.I.R. se répartissent comme suit :

$e e$ pour les mères n'ayant donné aucun enfant à duvet rayé ;

$e e^{+}$pour les mères ayant donné des enfants à duvet non rayé et d'autres avec une ou des rayures plus ou moins nettes;

$e^{+} e^{+}$pour les mères dont les enfants présentaient tous au moins des traces de rayures.

Ceci étant supposé, on trouve les fréquences suivantes, pour ces trois génotypes :

\section{TABLFAU $\mathrm{X}$}

Génotypes supposés des mères pour e et $\mathrm{e}^{+}$.

\begin{tabular}{|c|c|c|c|}
\hline & mères ce & mères $e^{+}$ & mères $\mathrm{e}^{+} \mathrm{e}^{+}$ \\
\hline sème test automne $\mathbf{s} 953 \ldots$ & IO & 24 & I \\
\hline Printemps 1954 $\ldots \ldots \ldots \ldots$ & 18 & 20 & — \\
\hline Total ......... & 28 & +4 & I \\
\hline
\end{tabular}

Les fréquences de $e$ et $e^{+}$, estimées sur le total, sont :

$$
p_{e}=68 \% \quad q_{e^{+}}=32 \%
$$

I1 est d'ailleurs probable que la fréquence obtenue pour $e^{+}$est un 
minimum, la manifestation de $e^{+}$en présence de $e$ pouvant être pratiquement impossible à déceler.

I a dominance incomplète de $e^{+}$sur $e$ est indiquée par les proportions respectives d'animaux à duvet non rayé et de ceux présentant au moins des traces de rayures, chez les mères ayant des descendants dans les deux catégories.

TABLEAU XI

\begin{tabular}{|c|c|c|}
\hline & Duvet non rayé & Duvet à rayures plus ou moins \\
\hline Ier test aut... & $5 \circ$ & 22 \\
\hline $2^{\mathrm{e}}$ test aut $\ldots \ldots$ & 168 & 104 \\
\hline Print. I $954 \ldots \ldots \ldots \ldots \ldots \ldots$ & r Io & 104 \\
\hline 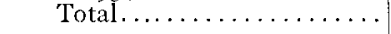 & 328 & $23^{\circ}$ \\
\hline
\end{tabular}

En F2, les parents qui avaient été supposés tous deux $e e$ ont donné des enfants à duvet non rayé, sauf parfois avec une tache sur le crâne ou une trace de rayure, en général beaucoup plus estompée qu'en FI. Ceci paraît néanmoins confirmer que certains parents à duvet exempt de toute trace de rayure devaient être hétérozygotes $e e^{+}$, et est à rapprocher du défaut de duvets à rayure ou trace de rayure trouvé en FI.

L,es croisements où l'un des deux parents présentait au moins une trace de rayure dans le duvet ont donné certains descendants également à rayure, parfois nette, souvent plus ou moins fortement estompée, mais toujours en proportion moindre que celle qu'on trouverait si $e^{+}$s'exprimait toujours en présence de $e$.

TABLEAU XII

\begin{tabular}{|c|c|c|}
\hline \multirow{2}{*}{$\begin{array}{c}\text { Génotype supposé } \\
\text { des parents }\end{array}$} & \multicolumn{2}{|c|}{ Enfants } \\
\hline & Duvet sans taches & Duvet à traces de rayures \\
\hline 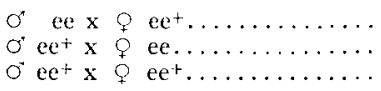 & $\begin{array}{r}267 \\
127 \\
12\end{array}$ & $\begin{array}{r}93 \\
96 \\
3\end{array}$ \\
\hline
\end{tabular}

Il a été mentionné plus haut que le duvet rayé ou à traces de rayures s'accompagne souvent d'un sous-plumage foncé dans la région dorsale. Cependant, la concordance n'est pas complète, comme le montrent les résultats suivants du printemps 1954 chez les $q$ employées ultérieurement comme reproductrices pour la F2 et dont le sous-plumage a été examiné attentivement ('Tableau XIII).

L'analyse d'une partie des résultats de $1955(\mathrm{~F} 2)$ montre de même une correspondance significative, mais non totale. (Tableau XIV) 
TABLEAU XIII

\begin{tabular}{|c|c|c|c|}
\hline & \multicolumn{3}{|c|}{ Duvet } \\
\hline & Rayé & traces de rayures & non rayé \\
\hline 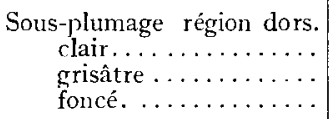 & $\begin{array}{r}1 \\
4 \\
17\end{array}$ & $\begin{array}{l}4 \\
5 \\
9\end{array}$ & $\begin{array}{r}54 \\
5 \\
4\end{array}$ \\
\hline
\end{tabular}

TABLEAU XIV

\begin{tabular}{l|c|c}
\hline & \multicolumn{2}{|c}{ Duvet } \\
\cline { 2 - 4 } & Rayure ou trace de rayure & non rayé \\
\hline Sous-plumage clair ............ & I7 & 294 \\
Sous-plumage grisâtre ou foncé. & 44 & 140 \\
\hline \hline
\end{tabular}

Il est donc probable que certains gènes agissent sur la couleur du duvet et non du sous-plumage des régions correspondantes, ou inversement. Un fait intéressant est que la majorité des duvets rayés s'accompagne d'un sous-plumage foncé, alors qu'inversement les sous-plumages foncés correspondent souvent à des duvets non rayés. Nous verrons plus loin que l'on peut peut-être rapprocher ce fait de la présence d'un facteur de " coloration retardée ".

Enfin, la comparaison de la proportion du duvet rayé chez les $O^{x}$ et chez les $q$ a donné les résultats suivants :

En FI, $249 \sigma^{x}$ à duvet non rayé, 43 à rayure plus ou moins nette. 206 q̀ duvet non rayé, 86 à rayure plus ou moins nette.

I a différence de proportion est significative. L'examen des génotypes des parents a montré qu'une explication par la présence de $B$ chez certains poussins $\sigma^{r}$ et non chez leurs sœurs ne suffisait probablement pas.

On pouvait encore, pour la $\mathrm{FI}$, rapporter la différence de proportion au fait que les $o^{x}$ étaient $S s$ et les $O s^{-}$. Cependant, la F2 prise globalement contenait des $\sigma^{x}$ et des $O[S]$ ou $[s]$. Or, on y trouvait :

$285 O^{x}$ à duvet non rayé, 90 à rayure plus ou moins nette,

219 O à duvet non rayé, I 40 à rayure plus ou moins nette.

Les familles de I955 (F2) où l'on observait une ségrégation pour le duvet rayé et d'où $B$ était absent ont été examinées pour la proportion du duvet rayé en fonction du sexe et de la présence de $S$ et $s$. Les résultats suivants ont été obtenus (Tableau XV)

Il y a donc davantage de duvets rayés chez les $q$ en l'absence de $B$, que $S$ ou $s$ soit présent. L'intensité de la rayure parait d'ailleurs plus 
TABleau XV

\begin{tabular}{|c|c|c|c|c|}
\hline & $\sigma \quad[S]$ & ? [S] & $\sigma^{\prime}[s]$ & $q[\mathrm{~s}]$ \\
\hline $\begin{array}{l}\text { Duvet non rayé } \ldots . \ldots \ldots \ldots \ldots \\
\text { Duvet à rayure pilus ou moins } \\
\text { nette } \ldots \ldots \ldots \ldots \ldots \ldots \ldots \ldots\end{array}$ & $\begin{array}{r}49 \\
60\end{array}$ & $\begin{array}{l}85 \\
62\end{array}$ & $\begin{array}{l}60 \\
\mathrm{r}_{4}\end{array}$ & $\begin{array}{l}77 \\
28\end{array}$ \\
\hline
\end{tabular}

grande en moyenne chez les $q$. Ceci est à rapprocher du fait connu que chez certaines races à duvet pigmenté, incluant la Rhode-Island, le duvet de la o est plus foncé que celui du ơ (6), (8), (27).

\section{5. - Le " blue ": G (ou Bl suivant Hutt (13)).}

Le "blue ", $G$, dilue à l'état hétérozygote le pigment noir et lui donne une teinte gris-bleu. Etudié pour la première fois par BATEson et PunNeTt (Igo6), il se trouve en particulier chez l'Andalouse bleue.

En FI, des plumages typiquement "bleus " ont été observés dans le premier test d'automne I953 sur des descendants à noir étendu, les duvets correspondants étant assez variables, du noir franc au gris.

Dans le deuxième test et au printemps 1954, où l'on n'obtenait aucun descendant à noir étendu, son observation à 8 semaines était plus difficile. Chez certains animaux, les zones du plumage possédant normalement du pigment noir, camail, grandes plumes des ailes et queue, étaient d'un gris plus ou moins clair, et, vu la similitude avec l'action observée chez les animaux à noir étendu, cette action a été également attribuée à $G$. Une douzaine de mères ont été reconnues $G g$; mais il est apparu que $G$ passait inaperçu chez un certain nombre d'animaux de type herminé possédant à 8 semaines une zone très réduite de pigment noir au camail et aux ailes. D'autre part, la teinte du "gris " ou "bleu " obtenu est variable et doit dépendre de facteurs secondaires modificateurs. Dans quelques cas relatés plus haut, du " bleu " très clair, peut-être parfois en présence de $B$, a tout d'abord été noté comme blanc dominant.

L, a proportion d'enfants "blue " et non "blue " chez les mères ayant les deux laisse d'ailleurs apparaître un défaut constant des premiers : 39 "blue "/59 non "blue " au printemps 1954 ; le \%" par rapport à la proportion I/I est 4 , I d'où $p<0,05$. I a génération suivante montre que certains parents ont eu des enfants porteurs du "blue ", alors qu'il n'avait pas été noté chez eux. La proportion des "blue " aux non "blue " dans les croisements $G g \times g g$ est ici au total de 102 "blue "/202 " non blue ", montrant encore un fort défaut des premiers.

Une estimation de la fréquence de $G$ chez les mères doit donc se limiter aux $q$ du $I^{\text {er }}$ test d'automne I953 ayant eu suffisamment d'en- 
fants à noir étendu : Io $q$ ont été trouvées $G g$, et 5 sûrement $g g$, ce qui donne une fréquence $p_{G}=33 \%$ et $q_{g}=67 \%$

\section{6. - Barrure liée au sexe : B.}

Le I er test d'automne 1953 n'était pas approprié pour analyser la présence de $B$ chez les $Q$ Gâtinaises. Cette présence n'a été examinée d'une façon systématique qu'à partir du printemps I954.

JAAP (I5) note que, chez les $\sigma^{x}$ de type herminé, on peut le plus souvent reconnaitre $B$ à Io semaines sur le camail, sauf chez quelquesuns où les plumes du camail n'ont pas assez de noir; chez les $O$, les plumes du cou et du camail sont souvent si claires qu'une classification exacte en $B$ et $b$ est phénotypiquement impossible.

Les observations faites ici ont porté sur des animaux un peu plus jeunes (8 semaines) et ont effectivement montré, d'une part que $B$ était présent chez nos Gâtinaises, d'autre part qu'il n'a pu être détecté chez tous les animaux qui le portaient. La barrure n'a été observée en FI que chez des $\sigma^{x}$, confirmant qu'il s'agissait bien d'un caractère lié au sexe et venant de certaines mères. Mais quelques-unes ont donné des fils manifestement barrés, et d'autres chez qui il était impossible de reconnaître nettement une barrure. Près de la moitié des $q$ se sont révélées $B^{-}$.

En F2 certains $\sigma^{r} B b$ ont été accouplés à des $q b$. Les proportions de descendants trouvés $B$ avec certitude ont été les suivantes:

chez les fils : 166 barrés/244 non barrés;

chez les filles : ro5 barrées/264 non barrées.

$B$ apparaît bien être plus difficile à repérer sur les $q$ herminées que sur les $\sigma^{x}$.

\section{7. - Patte jaune : w.}

$A u$ total, 8 poules, sur une centaine, analysées par les ơ R.I.R. ont été trouvées hétérozygotes $W w$.

A la génération suivante, une ségrégation a été observée, sur l'ensemble des croisements R.I.R. $\times$ Gâtinaise, entre $W$, apporté par la grande majorité des Gâtinaises, et w, apporté par les R.I.R.

HuTr (I3) note que la classification entre le phénotype "patte blanche " et. "patte jaune "peut être difficile jusqu'à 3 mois. Selon Collins, Thayer et Skoglund (36) le gène majeur pour la patte jaune est $w$, mais des gènes secondaires et des influences du milieu peuvent modifier quelque peu son expression chez les jeunes animaux.

La concordance entre le phénotype "pattes jaunes " et le génotype wro est apparue satisfaisante sur nos données, où nous avons observé 
à 8 semaines un type à pattes jaunes nettement distinct. Les proportions observées dans le total des croisements faits en automne I954 et au printemps 1955 sont les suivantes:

TABLEAU XVI

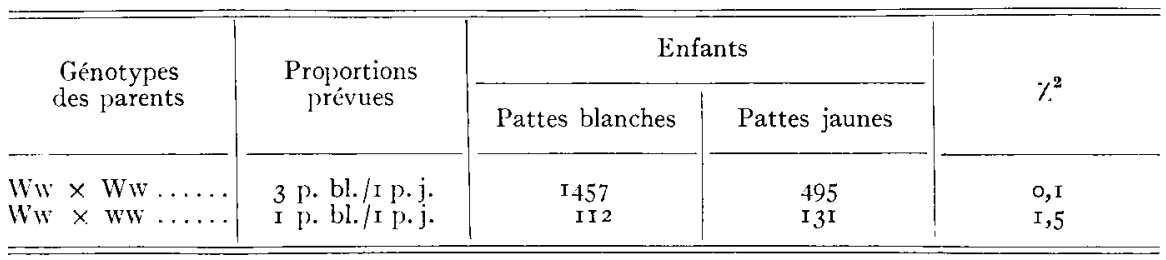

Ces proportions sont donc en très bon accord avec les proportions théoriques.

Cependant, une vingtaine des animaux classés " à pattes blanches ", observés de nouveau à Io semaines, se sont révélés présenter une teinte jaune pâle du bec. Quoique $W$ soit considéré en général comme complètement dominant, il sera intéressant de vérifier sur la génération suivante si ces animaux étaient des hétérozygotes $W w$ ou des homozygotes wre. Dans le premier cas, on pourrait éliminer sans progeny-test au moins une petite partie des hétérozygotes, indésirables puisque nous cherchons à fixer la patte blanche. Mais cette élimination ne serait sans doute que très partielle.

\section{8. - Emplumement lié au sexe.}

L.es $\sigma^{r}$ R.I.R. utilisés pour obtenir la Fi étaient tous homozygotes pour l'emplumement rapide lié au sexe $k k$. En FI, tous les coquelets apparaissaient à I jour avec l'emplumement lent, toutes les poulettes avec l'emplumement rapide, ce qui montre que toutes les $q$ Gâtinaises analysées étaient $K^{-}$.

Les coqs FI ont donné à la génération suivante 346 filles à emplumement lent/319 à emplumement rapide, conforme à la proportion théorique $\mathrm{I} / \mathrm{I}\left(\chi^{2}=\mathrm{I}, \mathrm{I}\right)$, ce qui confirme leur hétérozygotie.

\section{III. - OBSERVATIONS SUR CERTAINS PhéNOTYPES PARTICULIERS}

\section{1. - Pigmentation brune. Le "saumon ».}

De nombreuses observations font état d'une pigmentation brune ou rougeâtre, non confondue avec le "doré " qu'on sait être dû à $s$. AGAR (I), dans la Ire génération d'un croisement $\sigma^{x}$ Plymouth Rock 
barré $\times$ $\propto$ R.I.R., trouve des $0^{\top}$ portant des taches marron ("chesnut ") plutôt plus foncées que la couleur des R.I.R. surtout sur le dos et le dessus des ailes, très variables en étendue. La constitution génotypique de ces $\sigma^{x}$ devait être $E e$ Ss. Il trouve aussi ces taches brunes chez quelques $\sigma^{x}$ et $\odot$ issus du croisement $\sigma^{x} \mathrm{FI} \times q$ R.I.R.

GOODALE (II) remarque tne distribution analogue de ce pigment chez des L,eghorns dorés et chez des animaux noirs.

De nombreux génotypes semblent permettre cette pigmentation. AGAR la constate, non seulement chez des types "argenté ", mais aussi chez des ss, où elle peut être plus difficile à distinguer de la coloration générale. Cette pigmentation lui apparaît distincte du "doré ». Vu la variabilité de la teinte entre races rouges et parfois à l'intérieur d'une race, de nombreux auteurs ont noté que des gènes multiples devaient intervenir pour donner la couleur rouge. Ceci a été montré sur la Rhode Island rouge par WARREN et GORDON (35), HAYS (I2).

JULL (I8) et HUTT (I3) mentionnent du brun pouvant apparaittre en présence du blanc dominant.

Kimbali, (20) rapproche le pigment sur les ailes et le dos des $0^{x}$ du pigment plus clair (analogue au "saumon " de la Faverolles) apparaissant sur la poitrine des $\%$. $S$ n'empêche pas son apparition même sur le blanc récessif.

HuTT rapproche également (13) la teinte saumonée observée chez des $q$ Leghorn blanches d'une teinte correspondante, plus foncée, à la base des ailes des $\sigma^{T}$; il conclut que, dans le complexe non analysé de gènes autosomaux causant la couleur rouge, il $\mathrm{y}$ en a un ou plusieurs avec des effets restreints à la poitrine et au cou des $Q$, aux épaules et au dos des $\sigma^{x}$, visibles à des degrés divers, à la fois chez des animaux blancs et colorés.

D'après Kimbali, ces effets seraient dus à $e^{+}(20)$.

Des implantations d'hormones montrent encore la différence du " brun " avec le "doré ". Une implantation de "pellets " d'œstrogènes "blanchit " le brun montré par des $\sigma^{x}$ issus du croisement $\sigma^{x}$ R.I.R. $\times q$ Wyandotte blanche (7), de constitution génétique $C c S s e e^{+}$.

Nous distinguerons ici les observations relatives aux taches brunes des $\sigma^{x}$ et à la pigmentation brune ou saumon des $q$.

a) Pigmentation brune des $\sigma^{x}$.

Sur les données présentes, tous les $o^{t}$ non porteurs de blanc domi-

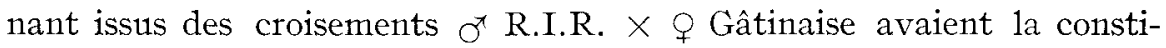
tution génétique $C c$ Ss. Sur I40 de ces animaux, des taches allant du jaune au brun-rouge, d'étendue et d'intensité variables, ont été notées sur le dos et la base des ailes; sur I36, il n'en a pas été observé. Ces taches n'excluaient pas l'observation éventuelle, par un examen attentif, de parties de plumes typiquement "dorées " dues selon toute vraisem- 
blance à une manifestation très partielle de $s$ malgré la présence de $S$.

A la génération suivante les ơ avec ou sans "brun "se répartissaient de la façon suivante :

TABLEAU XVII

\begin{tabular}{|c|c|c|}
\hline Génotype des o & Brun & sans brun \\
\hline SS $\begin{array}{ll}\text { Ss } \\
\text { ou } \\
\text { SS }\end{array}$ & $\begin{array}{c}\operatorname{I15} \\
27 \\
\text { I (?) (douteux) }\end{array}$ & $\begin{array}{r}300 \\
227 \\
49\end{array}$ \\
\hline
\end{tabular}

Ces résultats indiquent qu'une double dose de $S$ réduit fortement, ou peut-être empêche totalement la manifestation de ces taches brunes, tandis qu'une simple dose la permet : sauf un cas douteux, aucun $\sigma^{x}$ sûrement SS n'avait de taches brunes. On pouvait ici pratiquement déceler sans erreur, par l'apparence seule, le génotype $S s$ sur une fraction importante des $\sigma^{r}$, d'où la possibilité intéressante pour nous, d'une réduction notable du nombre de ces $\sigma^{x}$ à tester sur la descendance pour distinguer les $S s$ des S.S. Ceci est à rapprocher de la suggestion de Cock et PEASE (9) suivant lesquels $S$ ne supprime pas le " pile " chez les $q$ mais réduit probablement son apparition chez les $\sigma^{x}$.

Les rapports avec la présence de $e^{+}$sont moins nets ici. La liaison entre la présence de taches brunes sur le plumage et un duvet rayé ou à traces de rayures semble peu étroite. Une certaine corrélation a été observée avec un sous-plumage gris ou sombre, sur les $O^{x}$ en 1955 :

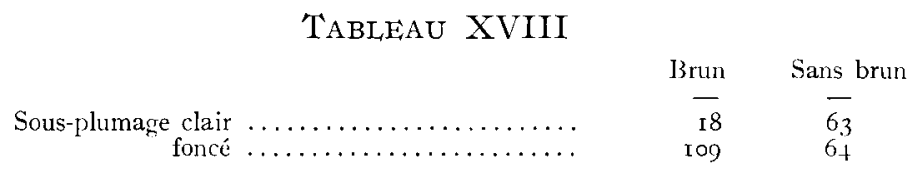

Si $e^{+}$favorise ou permet la présence de ces taches brunes, il est raisonnable de penser qu'il ne doit pas être seul en cause.

La même pigmentation a été observée, chez la moitié environ des coqs $I i S s$, ainsi que sur des $\sigma^{x}$ Ii ss.

b) Pigmentation brune ou saumon Des $q$.

En F2, les croisements de diverses sortes effectués faisaient apparaitre 24 \& $S$ (argentées) présentant une pigmentation moins foncée en général que le "brun " décrit précédemment chez des $\sigma^{x}$, rappelant chez certaines le "saumon".

De même, un saumon très pâle a été observé chez certaines $q$ à blanc récessif, et il a été montré que cette teinte ne devait pas être confondue avec une manifestation occasionnelle de $s$ (cf. p. rI).

D'autres recherches sont nécessaires pour apporter davantage de 
précisions sur les gènes en cause, et pour savoir si on peut attribuer en tout ou en partie la pigmentation des $\sigma^{x}$ et celle des $q$ à l'action des mêmes gènes.

\section{2. - Coloration retardée.}

PeAse et Cock (28) relatent, chez des animaux dorés et portant la barrure liée au sexe, la présence d'un gène autosomal récessif produisant un duvet blanc, ou une " rayure réduite ". Selon eux, le duvet herminé du type Sussex pourrait être un cas de duvet "retardé ".

Kimbali (I9), (22) invoque l'existence d'un gène Wh (Wheaten) présent chez le "Wheaten Game " et la Faverolles, produisant à l'état homozygote un duvet blanc, à l'état hétérozygote en présence de $e^{+}$un duvet avec une seule bande marron non encadrée de deux autres.

Lors du croisement des $q$ Gâtinaises avec des $\sigma^{\top} E e$ ( ${ }^{\text {er }}$ test d'automne I953) des poussins à duvet blanc ont pris par la suite un plumage coloré :

I9 sont devenus noirs,

20 ont pris un plumage à noir incomplètement étendu,

9 ont pris un plumage rouge.

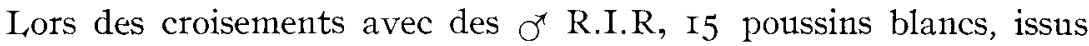
de Io mères différentes, ont pris à 8 semaines un plumage rouge.

La présence d'un facteur de coloration retardée est donc manifeste. Touchant son origine et son mode de transmission génétique, des recherches ultérieures sont nécessaires, incluant l'examen de la descendance d'animaux ayant présenté ce caractère, pour la coloration du duvet et l'extension éventuelle des rayures (stripe). Il est possible qu'il s'agisse du facteur $W h$ de Kimball, et que des "stripe " réduits aient été causés par lui. Ceci pourrait être rapproché du manque de concordance entre duvet et sous-plumage de la région dorsale, la majorité des duvets rayés s'accompagnant d'un sous-plumage foncé, alors que tous les sous-plumages plus ou moins foncés sont loin de correspondre à des duvets "rayés".

Des croisements avec des Faverolles pourraient donner des indications utiles.

D'autres effets faisant penser à une coloration retardée ont été observés. Une mère Gâtinaise a donné, outre une fille à duvet blanc et à plumage rouge herminé, 3 fils et I fille à plumage rouge herminé et à duvet rouge pâle, portant des rayures blanches à l'emplacement ordinaire des bandes du duvet "stripe ». Ces animaux n'ont révélé ni la présence de $G$, ni celle de $B$, pouvant atténuer la pigmentation noire. Un autre facteur paraît donc avoir empêché totalement la pigmentation sombre des rayures et sans doute partiellement celle du duvet. 
A la génération suivante (automne I954-printemps I955) des cas de coloration retardée ont encore été observés. I7 animaux à duvet blanc ont donné des plumages rouges à 8 semaines. D'autres animaux, apparemment non barrés, ont présenté encore des duvets rouges en général assez pâles avec des rayures blanches.

Peu des animaux qui présentaient en FI une coloration retardée ont reproduit. Une $q$ qui présentait les rayures blanches sur fond rouge pâle dans le duvet a donné 4 filles à coloration retardée (duvet blanc, plumage rouge) et les autres enfants à duvet rouge pâle. Une autre mère quí présentait les mêmes caractères a donné des enfants d'apparence normale.

Toujours dans le but de chercher les expressions possibles de la " coloration retardée ", la pigmentation des rémiges primaires à I jour a été observée en I955. C'est un fait connu, rappelé par Huт'т (I3) que, chez les animaux ne portant ni le blanc dominant, ni le blanc récessif, les primaires ont en général à la naissance du pigment noir. Cependant, ceci n'est pas constant. L'absence de pigment noir est-elle due à des causes accidentelles ou au génotype du poussin, et, dans ce cas, peut-on mettre ceci sur le compte du même facteur de "coloration retardée "?

Nous avons toujours trouvé les primaires non pigmentées chez les I $i$ ou les $c c$. Chez les animaux colorés, 349 ont été notés sans pigmentation décelable dans les primaires, II 38 avec pigmentation, la plupart des mères ayant à la fois des enfants dans les 2 catégories. Ceci ne semble pas présenter de rapports avec la présence de divers gènes de coloration : $B, G, C C$ ou $C c, S, s$, ni avec le sexe. Si ce caractère présente une base génétique, il n'a pas non plus de rapport apparent avec la coloration retardée consistant dans la suppression sur le duvet du pigment rouge ; car les animaux ayant ce dernier caractère en 1955 avaient pour la majorité du pigment noir dans les rémiges à I jour (I4 sur I7).

\section{3. - Patte grise ou bleutée.}

L'action de $i d$, lié au sexe, produisant la patte bleue, est sujette à l'action de gènes modificateurs, tel $E$, renforçant la pigmentation, $I$, $c$ et $B$, la diminuant, suivant HUtT (I3), StURkie (33), JefFrey (I6). SkALLER et Grigg (3I) font appel à 4 gènes autosomaux et 2 liés au sexe.

KRALLINGER (24) trouve que le pigment hypodermique des pattes est inhibé, chez la Leghorn par un gène dominant lié au sexe, chez la Faverolles par des gènes modificateurs autosomaux.

JAAP (I4) rencontre des pattes "satle " ("Willow )) chez des Plymouth Rock blanches $c c$ ou $I$, empêchées par $B$, ou $I d$ lié à $B$. Il en trouve également chez la race Delaware, de type herminé (I5). Il observe davantage de pattes grises en l'absence de $B$, et davantage chez les $q$ que 
chez les $\sigma^{\top}$. Selon, lui $i d$ n'explique pas ces pattes grises qui sont d'autre part sujettes à d'autres gènes modificateurs que $B$.

Dans notre FI ( $\sigma^{\top}$ R.I.R. $\times q$ Gâtinaise) sont apparues $2 q$ dont les pattes étaient bleutées, issues de 2 mères Gâtinaises.

En F2, des pattes bleutées ou d'un gris assez clair sont apparues en plus grand nombre, à l'automne I954, puis au printemps 1955, où les résultats suivants ont été notés :

Dans la descendance des mères ayant au moins un enfant à pattes bleutées, on trouve :

chez les fils, 3I à pattes bleutées/48I à pattes non bleutées;

chez les filles, I36 à pattes bleutées/42I à pattes non bleutées.

Une partie seulement de ces mères avaient des fils à pattes bleutées (25 au total); dans leur descendance, on trouve :

chez les fils, 3 I à pattes bleutées/206 à pattes non bleutées;

chez les filles, 5I à pattes bleutées/I70 à pattes non bleutées.

Dans les deux cas, il est intéressant de noter que les proportions chez les filles s'accordent bien avec la proportion théorique $3 / \mathrm{I}$, autrement dit avec 1'hypothèse de la ségrégation $\mathrm{F}_{2}$ d'un gène dont l'allèle récessif autosomal permettrait la patte bleutée $\left(\gamma^{2}=0\right.$, I dans le I ${ }^{\text {er }}$ cas ; $\%^{2}=0,4$ dans le $2^{\mathrm{e}}$ cas).

Par contre, la proportion des fils à pattes bleutées prise dans les familles en comportant ne s'accorderait qu'avec l'hypothèse d'une proportion 7 pattes non bleutées/I patte bleutée $\left(\%^{2}=0, \mathrm{I}\right)$ et la proportion des fils à pattes bleutées prise dans toutes les familles dont au moins un enfant présente ce caractère se rapproche de 15 pattes non bleutées/ $\mathrm{I}$ patte bleutée $\left(\%_{2}=0,0\right)$. La présence de plusieurs gènes est donc certainement nécessaire à la manifestation de la patte bleutée chez les coqs en $\mathrm{F} 2$.

Des résultats ultérieurs sont nécessaires pour préciser la nature des gènes en cause. On peut seulement noter pour l'instant que les gènes $C, I, B, G, S, e$ ou $e^{+}$, n'ont pas eu d'influence nette sur l'apparition de la patte bleutée en $\mathbf{F} 2$.

\section{IV. - CONCLUSIONS}

\section{1. - Génotype de la Gâtinaise.}

Pour nous résumer, nous avons trouvé, sous le blanc récessif que possédaient toutes nos Gâtinaises, les cryptomères suivants :

Avec une fréquence notable, le blanc dominant $I$, l'allèle $e^{+}$de la série " extension du noir ", le "bleu " $G$, la barrure liée au sexe $B$, concurremment avec les allèles $i, e, g, b$; plus rarement, le doré lié au sexe $s$.

Le noir étendu $E$ n'a pas été trouvé. 
Le récessif $w$ (pattes jaunes) est rare, et l'emplumement lent lié au sexe $K$, semble seul exister.

Une pigmentation brune des $\sigma^{x}$, une pigmentation plus claire des ${ }^{\prime}$, des cas de coloration retardée et de pigmentation dermique des pattes, ont été mis en évidence en croisement, leur conditionnement génotypique restant à préciser.

I,es Gâtinaises élevées à Jouy provenaient, à l'origine, de trois élevages situés dans des régions différentes. Nous avons pu nous assurer par l'examen des pedigrees des poules analysées, que le blanc dominant en particulier, était présent dans les trois souches d'origine. Il semble donc que les mêmes cryptomères existent chez des populations de provenances diverses. Il paraît assez plausible que la Gâtinaise ait subi, dans un passé plus ou moins éloigné, quelques croisements faits dans un but améliorateur ( $c f$. P. CHARLET, B.T.I. I952, no 66). La présence de $I$ et $w$ fait penser à la Leghorn, peut-être à la Wyandotte.

\section{2. - Programme de sélection relatif aux gènes étudiés.}

I'analyse nous permet d'orienter le programme de sélection de notre souche de Gâtinaises pour les allèles que nous venons d'étudier. La souche doit être homogénéisée pour certains cryptomères, afin d'obtenir, avec des races non blanches, des croisements uniformes, et, éventuellement, un sexage à la naissance. Le même problème doit vraisemblablement se poser à tous les éleveurs de Gâtinaises voulant par exemple obtenir des croisements pour le poulet de chair.

D'autre part, il va de soi que, moins on éliminera d'animaux sur des gènes de plumage, plus la sélection exercée sur les caractères de production pourra être forte. Aussi garderons-nous, chaque fois que nous le pourrons, l'allèle ayant la plus grande fréquence.

Nous éliminerons donc $I$, allèle moins répandu que $i$, et plus facile à supprimer parce que dominant. Nous perdrons ainsi l'avantage d'avoir des croisements de première génération blancs (avantage pour le poulet de chair), mais nous réserverons par contre la possibilité de sexage par $B$, en présence de $e^{+}$ou $E$.

Il pourra s'avérer intéressant d'étendre à toute la souche les allèles $e^{+}$et $B$, déjà présents en proportion notable, et qui permettraient, dans certains croisements, un sexage à la naissance. Dans cette éventualité, il serait nécessaire d'étudier d'une façon plus complète et d'éliminer le facteur de coloration retardée manifesté chez certains sujets. Quant à $G$, il n'a pas d'action sur la couleur rouge, donc n'empêche pas le sexage par $s$. La question se posera de savoir s'il peut être gênant dans des croisements avec une race $E$, par exemple, pour le sexage par $B$.

Enfin, la souche doit être complètement pure pour la patte blanche, 
de façon à avoir des croisements de première génération uniformes à pattes blanches avec des races à pattes jaunes.

Tel est le programme de sélection qui se présentait à première vue pour nos Gâtinaises. Mais d'autres considérations nous ont conduits à envisager le croisement avec des Rhode Island rouges. Notre souche de Gâtinaises étant plutôt tune souche de chair, l'introduction de R.I.R. devrait améliorer ses aptitudes à la ponte. Nous pourrons d'autre part répandre partout l'allèle $s$, et ce rouge cryptomérique permettra certains croisements sexables. De plus, la souche de R.I.R. employée apportera l'emplumement rapide $k$, totalement absent chez nos Gâtinaises.

Pour conclure, insistons sur le fait qu'en dehors des possibilités de sexage à la naissance, l'homogénéité des croisements de première génération obtenus à partir de deux races pures est d'une importance commerciale notable. La plupart des contestations qui s'élèvent entre vendeurs et acheteurs de poussins de croisement sur la pureté des races parentes proviennent du fait que les individus parents possédaient une grande variabilité génotypique, masquée en race pure par un blanc dominant ou récessif (Bresse, Wyandotte, Gâtinaise...) ou encore par du noir (Marans). L'obtention de souches à gènes cryptomères connus, et homozygotes pour ces gènes, est donc nécessaire, et il est probable que cette tâche aurait déjà été accomplie par les éleveurs s'ils avaient pu ou su la réaliser d'une façon simple.

La fréquence de tels cas d'hétérogénéité cachée en race pure, mais se manifestant en croisement, fait une fois de plus mesurer combien la notion de race pure définie d'après un standard uniquement descriptif est une notion incomplète. En suivant l'orientation indiquée par LusH (37) qui propose d'établir des standards de races basés sur une formule génétique, les expositions et les jugements d'animaux sur l'apparence extérieure pourraient avoir, en dehors de leur aspect "sportif ", un rôle de guide pour les sélectionneurs qui s'attachent avant tout au point de vue économique de l'élevage.

\section{RESUME}

L'analyse génétique de poules Gâtinaises provenant de plusieurs souches différentes, faite dans le cadre d'un programme de travail plus étendu, a montré que, sous le blanc récessif $c$ universellement répandu, se trouvaient avec une fréquence notable le blanc dominant $I$, l'allèle $e^{+}$ de la série "extension du noir ", le "bleu " $G$, la barrure liée au sexe $B$, concurremment avec les allèles correspondants $i, e, g, b$; le doré lié au sexe $s$ et le récessif $w$ (pattes jaunes) sont rares. Le noir étendu n'a pas été trouvé, non plus que l'emplumement rapide lié au sexe $k$.

Quelques phénotypes particuliers ont été observés, incluant la mani- 
festation du doré en présence du blanc récessif, la pigmentation brune ou rappelant le "saumon ", une " coloration retardée " du plumage, et l'apparition en $\mathrm{F}_{2}$ d'une patte gris-bleuté. L'analyse de certains de ces caractères nécessite des observations ultérieures.

$$
\text { (Reçu pour publication le } 26 \text { décembre 1955). }
$$

\section{REFERENCES BIBLIOGRAPHIQUES}

(I) AGAR (W. E.). - Experiments with certain plumage colour and pattern factors in poultry. $J$. Genet., 14, 265-272, I924.

(2) Barrows (H. R.). - The histological basis of the different shank colors in the domestic fowl. Maine Agri. Exp. Sta. Bull., 232, IgI4.

(3) BERNiER (P. E.) et CoONEy (W. T.). - Black down color and riboflavin deficiency in embryos of the domestic fowl. Xe Congrès Mondial d'Aviculture, Section A, 66-7I, I954.

(4) Boyer (J. P.). - Étude génétique du plumage de la Marans coucou argentée. Annales Zoot., $4^{\mathrm{e}}$ année, 1, 93-102, I955.

(5) Caridroit (F.) et Regnier (V.). - L'analyse génétique de la poule domestique. La Revue Scientifique, 79, 4, 223-245, I94I.

(6) Carr (J. G.). - Autosexing Brown Leghorns. Autos. Annual, 5-7, I952.

(7) CoChEZ (L. P.). - Communications personnelles. I95I-I954.

(8) Cock (A.). - Autosexing without barring. Autos. Annual, 6-8, 1953.

(9) Cocr (A.) et PEASE (M.). - - La génétique du dessin pile blanc sur la volaille. IX Congrès Mondial d'Aviculture, vol. I, 47-5I, I95I.

(IO) CREW (F. A. E.). - Unexpected results of matings involving sex-linked characters. Atti del congr. mond. pollicoltura, 5 congr., 2, 223-226, I933.

(II) Goodale (H. D.). - Salmon Blacks. Anat. Record, 34, I7I-I72, I926.

(r2) Hays (F. A.). - Crossing production and exhibition Rhode Island Reds. Mass. Agr. Exp. Sta. Bul., 316, I935.

(I3) Hutr (F. B.). - Genetics of the fowl. Mc. Graw Hill Book Co N. Y., Ire éd. 590 p., I949.

(I4) JAAP (R. G.). - Strains of white plymouth Rocks for specific economic purposes. Poul. Sci., 22, 3, 209-2 I7, I943.

(I5) JAAP (R. G.). - Shank color and barred plumage in Columbian-colored chickens. Poul. Sci., 34, 2, 389-395, I955.

(I6) JEFFREY (F. P.). - Plumage color genes in white Plymouth Rock and White Wyandottes. Poul. Sci., 26, 5, 526-528, I947.

(I7) JÉrome (F. N.), Cavers (J. R.). - Crosses involving dominant and recessive white in combination with colors and patterns. Poul. Sci., 31, 3, 427-432, I952.

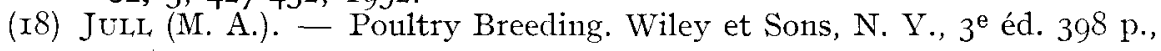
I952.

(Ig) Kimbal, (E.). - Wild type plumage pattern in the fowl. Jour. of Hered., 43, 3, I29-I32, 1952 .

(20) Krmbali (E.). - Genetic relation of extended black to wild type plumage pattern in the fowl. Poul. Sci., 31, I, 73-78, I952.

(2I) Kimbai. (E.). - Genetics of secondary plumage patterns in the fowl. Poul. Sci., 32, I, I3-17, 1953 .

(22) Kimbali (E.). - Genetics of buttercup plumage pattern in the fowl. Poul. Sci., 32, 4, 683-692, I953.

(23) Kimball (E.). - Genetics of birchen plumage pattern in the fowl. Poul. Sci., 33, 3, 472-48I, I954.

(24) Kralinger (H. F.). - Une étude mendélienne de 6 caractères morpho- 
logiques dans des croisements entre Leghorns et Faverolles. Ve Congrès Mondial d'Aviculture, vol. II, 380-393, I933.

(25) LEgrand (P.). - Contribution à l'étude de la couleur blanche du plumage chez les Poules. Bull. Biol. France Belg., 73, 433-437, I939.

(26) Lippincot'T (W. A.). - Further data on the inheritance of "Blue " in poultry. Amer. Nat., 55, 289-327, I92I.

(27) Mc Arthur (J. W.), Mc Ilraith (J. J.). - Color sexing of day-old Brown Leghorns. Poul. Sci., 25, 2, I80-183, I946.

(28) PEASE (M.) et Cock (A.). - Coloration retardée, un gène récessif affectant la couleur et le dessin du duvet. IXe Congrès Mondial d'Aviculture, vol. 1, 67-7I, I936.

(29) QUinn (J. P.). - Genes for color and plumage pattern in white varieties of chickens unsmarked by crossbreeding. Poul. Sci., 15, 2, I69-I78, I936.

(30) Rit'terhaus (T.). - Die Erbfaktoren der Dunenzeichnung der Haushiihner. Züchter 2, 324-330, I930.

(3I) Skaller (F.) et GrigG (G. W.). - The inheritance of shank colour in S. C. White Leghorn and Australorp fowls. Austr. Jour. Agr. Res., 2, 494-497, I95I.

(32) Smy'th (J. R.) et Bohren (B. B.). - A multiple allelic series affecting feather color in the domestic fow1. Poul. Sci., 28, 5, 782, I949.

(33) Sturkie (P. D.), Godbey (C. B.) et Sherwood (R. M.). - The inheritance of shank color in chickens. Poul. Sci., 16, 3, I83-I88, I937.

(34) WARREN (D. C.). - The inheritance of two standard disqualifications in white Leghorn chickens. Poul. Sci., 9, 5, 27I-282, I930.

(35) Warren (D. C.) et Gordon (C. D.). - Plumage and eye color inheritance in the single comb Rhode Island Red fowl. J. Agr. Res., 47, 8979I0, I933.

(36) Collins (W. M.), Thayer (S. C.) et Skoglund (W. C.). - Breed and strain differences in shank pigmentation in growing chickens. Poul. Sci., 34, I, 223-228, I955.

(37) Lush (J. L.). - Animal Breeding Plans. Iowa State Coll. Press. $3^{\mathrm{e}}$ éd, 443 p., I949. 